\title{
Two energy scales and slow crossover in $\mathrm{YbAl}_{3}$
}

\author{
A. L. Cornelius, ${ }^{1}$ J. M. Lawrence, ${ }^{2, *}$ T. Ebihara, ${ }^{3}$ P. S. Riseborough, ${ }^{4}$ C. H. Booth, ${ }^{5}$ M. F. Hundley, ${ }^{6}$ P. \\ G. Pagliuso, ${ }^{6}$ J. L. Sarrao, ${ }^{6}$ J. D. Thompson, ${ }^{6}$ M. H. Jung, ${ }^{7}$ A. H. Lacerda, ${ }^{7}$ and G. H. Kwei ${ }^{8}$ \\ ${ }^{1}$ University of Nevada, Las Vegas, NV 89154 \\ ${ }^{2}$ University of California, Irvine, CA 92697 \\ ${ }^{3}$ Shizuoka University, Shizuoka 422-8529, Japan \\ ${ }^{4}$ Temple University, Philadelphia, PA 19122 \\ ${ }^{5}$ Lawrence Berkeley National Laboratory, Berkeley, CA 94720 \\ ${ }^{6}$ Los Alamos National Laboratory, Los Alamos, NM 87545 \\ ${ }^{7}$ National High Magnetic Field Laboratory, Los Alamos National Laboratory, Los Alamos NM 87545 \\ ${ }^{8}$ Lawrence Livermore National Laboratory, Livermore CA 94551
}

(Dated: January 23, 2002)

\begin{abstract}
Experimental results for the susceptibility, magnetization, specific heat, $4 f$ occupation number, Hall effect and magnetoresistance for single crystals of the intermediate valence (IV) compound $\mathrm{YbAl}_{3}$ show that, in addition to the Kondo temperature scale $k_{B} T_{K} \sim 670 \mathrm{~K}$, there is a low temperature scale $T_{c o h} \sim 30-40 \mathrm{~K}$ for the onset of Fermi liquid coherence. Furthermore the crossover from the low temperature Fermi liquid regime to the high temperature local moment regime is slower than predicted by the Anderson impurity model. We discuss these effects in terms of the theory of the Anderson lattice and suggest that they are generic for IV compounds.
\end{abstract}

PACS numbers: 75.30.Mb 75.20.Hr 71.27.+a 71.28.+d 61.10.Ht

The low temperature transport behavior of periodic intermediate valent (IV) and heavy fermion (HF) compounds[1] is fundamentally different from that expected for the Anderson impurity model (AIM), in that it manifests vanishing resistivity (Bloch's law) and an optical conductivity[2] appropriate for renormalized band behavior. Similarly, the $4 f$ electrons have a coherent effect on the Fermi surface, as seen in de Haas van Alphen (dHvA) measurements[3]. However the temperature dependence of the susceptibility, specific heat and $4 f$ occupation number and the energy dependence of the dynamic susceptibility show behavior that is qualitatively very similar to the predictions of the $\operatorname{AIM}[4,5]$. Essentially this is because these properties are dominated by spin/valence fluctuations that are highly local and which exhibit a Lorentzian power spectrum[6] consistent with the AIM.

In this paper we report data for the IV compound $\mathrm{YbAl}_{3}$ and show that these latter properties can differ in at least two ways from the predictions of the AIM. First, the crossover from low temperature Fermi liquid behavior to high temperature local moment behavior is slower (i.e. more gradual) than predicted for the AIM. Second, anomalies (relative the AIM) in the magnetization, susceptibility, specific heat and magnetotransport occur below $30-40 \mathrm{~K}$, which is the scale $T_{\text {coh }}$ for the onset of coherent Fermi liquid $T^{2}$ behavior in the resistivity. We then argue that the existence of a slow crossover and a low temperature scale that is an order of magnitude smaller than the AIM Kondo temperature $\left(T_{c o h}<<T_{K}\right)$ may be generic features of IV compounds and of the behavior of the Anderson lattice.

The samples were single crystals of $\mathrm{YbAl}_{3}$ and $\mathrm{LuAl}_{3}$ grown by the "self-flux" method in excess Al. The sus- ceptibility was measured using a SQUID magnetometer and the specific heat was measured via a relaxation technique. The Hall coefficient was measured in a magnetic field of $1 \mathrm{~T}$ using an a.c. resistance bridge. The magnetoresistance was measured at the Los Alamos Pulsed Field Facility of the National High Magnetic Field Laboratory using a $20 \mathrm{~T}$ superconducting magnet and an a.c. bridge. The $4 f$ occupation number $n_{f}(T)$ was determined from the $\mathrm{Yb} L_{3} \mathrm{x}$-ray absorption near-edge structure, measured at the Stanford Synchrotron Radiation Laboratory (SSRL) on beam line 4-3; the technique for extracting $n_{f}$ from the data and other experimental details were similar to those discussed earlier [7]. We note here that the $\mathrm{Lu} L_{3}$ near-edge structure measured for $\mathrm{LuAl}_{3}$ was used as a standard.

In Fig. 1 we plot the susceptibility $\chi(T)$ and the linear coefficient of the $4 f$ specific heat, where $\gamma_{m}=C_{m} / T$ and $C_{m}=C\left(\mathrm{YbAl}_{3}\right)-C\left(\mathrm{LuAl}_{3}\right)$. (For $\mathrm{LuAl}_{3}$ at low temperatures, $C=\gamma T+\beta T^{2}$ with $\gamma=4 \mathrm{~mJ} / \mathrm{mol}-\mathrm{K}^{2}$ and $\beta=1.15 \times 10^{-4} \mathrm{~J} / \mathrm{mol}-\mathrm{K}^{4}$, which implies a Debye temperature $\Theta_{D}=257 \mathrm{~K}$.) The broad peaks near $100 \mathrm{~K}$ are typical of $\mathrm{Yb}$ IV compounds with $T_{K}$ greater than 500K (see Fig. 4). However, the low temperature specific heat coefficient displays an upturn below $30 \mathrm{~K}$ which saturates at $T=0$. In addition, the susceptibility increases below $40 \mathrm{~K}$ to a peak at $15 \mathrm{~K}$. This was reported earlier by Hiess et al.[8] who raised the possibility that it might represent an extrinsic effect due to antiferromagnetic short range ordering of $\mathrm{Yb}^{3+}$ impurities. Our argument against this is that the quality of our samples, as measured by the residual resistivity $(0.5 \mu \Omega-\mathrm{cm})$, is sufficiently high that $\mathrm{dHvA}$ signals are well-resolved[9]; the presence of $\mathrm{Yb}^{3+} \mathrm{impu-}$ rities should lead to a large residual resistivity. Furthermore, we find that as the residual resistivity increases, 


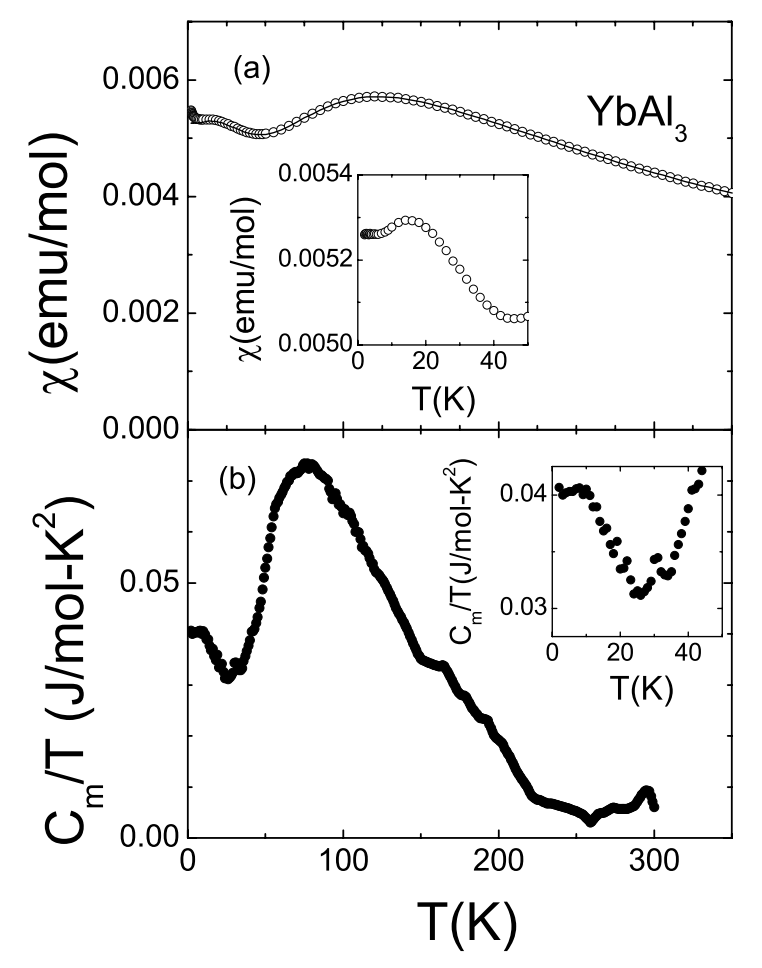

FIG. 1: (a) The magnetic susceptibility $\chi(T)$ and (b) the magnetic contribution to the specific heat coefficient $C_{m} / T$ for $\mathrm{YbAl}_{3}$. The insets exhibit the low temperature behavior; a small Curie tail has been subtracted from the data in the inset for $\chi(T)$.

the $T=0$ specific heat maximum decreases and a Curie tail grows in the susceptibility. Hence we associate the low temperature anomalies in susceptibility and specific heat with the purest samples, and assert that they are intrinsic. These anomalies are the basic evidence for the existence of a low temperature scale, $T_{c o h} \sim 30-40 \mathrm{~K}$ below which there is a significant change in the behavior of the compound.

In Fig. 2a we plot the magnetization versus applied magnetic field at low and high temperature; the solid lines are linear fits to the data. The difference between the linear fits and the data is shown in the inset. At $250 \mathrm{~K}$ the magnetization is linear in field up to $60 \mathrm{~K}$; at $4 \mathrm{~K}$ the data is linear up to $40 \mathrm{~T}$, above which there is a clear change in slope. We plot the slope $\chi(H)$ for low and high field versus temperature in Fig. 2b. The low field results compare well with the susceptibility measured for $\mathrm{B}=1 \mathrm{~T}$ in the SQUID magnetometer, showing a maximum near $125 \mathrm{~K}$ and a second maximum near $\mathrm{T}=0$. In the high field data, the low temperature maximum is absent and the data exhibit the qualitative features expected for an Anderson impurity (see, e.g., Fig. 4b). Clearly, the low temperature susceptibility anomaly is suppressed

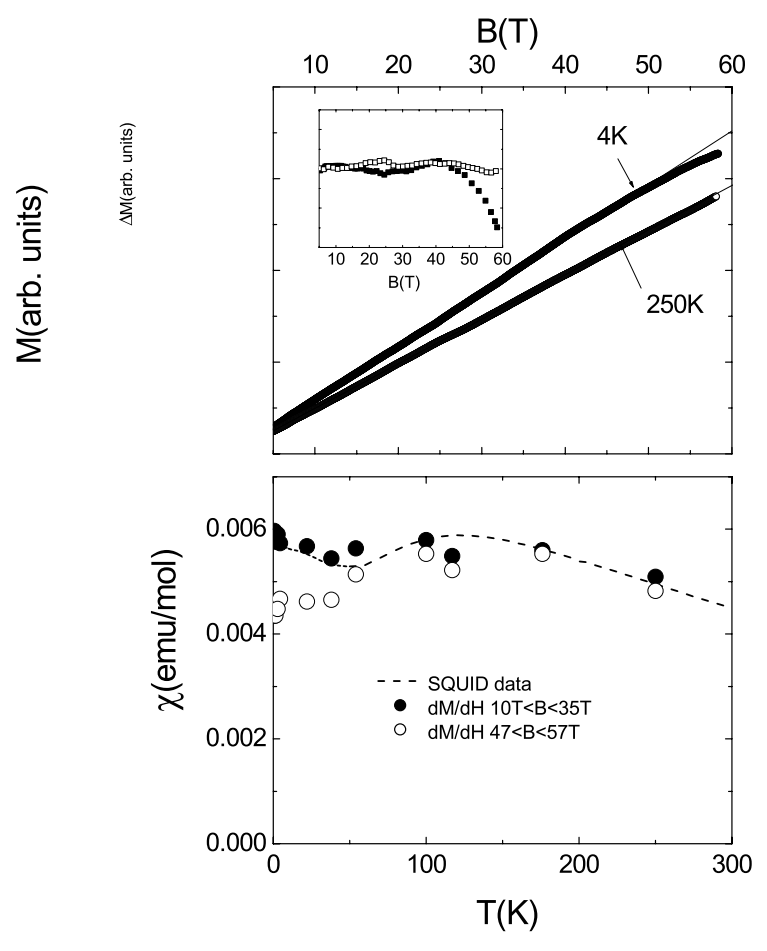

FIG. 2: (a) The magnetization $\mathrm{M}(\mathrm{H})$ versus the magnetic field at $4 \mathrm{~K}$ and $250 \mathrm{~K}$; the solid lines are linear fits to the data in the field range $10 \mathrm{~T}<B<35 \mathrm{~T}$. Inset: The difference $\Delta M$ between the data and the linear fits. (b) The susceptibility $\chi(T)$ measured at both low $(10 \mathrm{~T}<B<35 \mathrm{~T})$ and high $(47 \mathrm{~T}$ $<B<57 \mathrm{~T})$ magnetic field. The dashed line is data taken at $\mathrm{B}=1 \mathrm{~T}$.

by magnetic fields greater than $\mathrm{B}^{*} \sim 40 \mathrm{~T}$. Since $\mu_{B} B^{*}$ is of the same order as $k_{B} T_{c o h}$, this effect gives strong confirming evidence for the existence of a new low energy scale $k_{B} T_{c o h} \sim 3-4 m e V$ which is an order of magnitude smaller than the AIM Kondo scale. The inset of Fig. 3 shows that a $T^{2}$ behavior of the resistivity sets in below $30 \mathrm{~K}$, which makes it clear that $T_{c o h}$ is the temperature scale for the onset of coherent Fermi liquid behavior.

Evidence for the change of character can also be seen in the magnetotransport (Fig. 3). The Hall coefficient of $\mathrm{LuAl}_{3}$ is temperature independent, as is typical of a metal. The high temperature Hall coefficient of $\mathrm{YbAl}_{3}$ varies with temperature in a manner suggestive of scattering from $\mathrm{Yb}$ moments (although the data cannot be fit well with the standard skew scattering formula[10]). Near $50 \mathrm{~K}$ the derivative $d R_{H} / d T$ of the Hall coefficient changes sign. The magnetoresistance (Fig. 3b, inset) follows a $B^{2}$ law above $50 \mathrm{~K}$ and the magnitude is approximately the same for field parallel and transverse to the current. Below 50K, the magnetoresistance becomes more nearly linear and the transverse magnetoresistance becomes substantially larger $(\Delta R / R \sim 0.75)$ than the parallel magnetoresistance $(\Delta R / R \sim 0.35)$ at $2 \mathrm{~K}$. In Fig. 
3b we plot $\Delta R / R$ versus $B r_{0}$ where $r_{0}=R(150 K) / R(T)$; this tests Kohler's rule, i.e. that at any temperature $\Delta R / R=A f\left(B r_{0}\right)$ depends only on the product $B r_{0}$. The data violate this rule essentially because $\mathrm{A}$ varies with $\mathrm{T}$, increasing by a factor of almost 1.5 between 40 and $80 \mathrm{~K}$; this crossover is seen most clearly in the data measured as a function of temperature at a fixed field 17.5T. These magnetotransport anomalies suggest that the anomalies in $\chi$ and $C / T$ may be associated with an alteration of the Fermi surface.

To demonstrate that the crossover from Fermi liquid behavior to local moment behavior is slower than predicted by the AIM we proceed as in our recent paper on $\mathrm{YbXCu}_{4}$ [5]. The calculations were performed using the non-crossing approximation (NCA); the values of the AIM parameters are given in Fig.4. It is clear from Fig. 4 that the susceptibility, $4 f$ occupation number and $4 f$ entropy $S_{m}=\int d T C_{m} / T$ all qualitatively follow the predictions of the AIM. The calculated coefficient of specific heat $\left(\gamma=47.8 \mathrm{~mJ} / \mathrm{mol}^{-\mathrm{K}^{2}}\right)$ is within 20 of the measured value $\left(\gamma_{m}=40.65 \mathrm{~mJ} / \mathrm{mol}^{2} \mathrm{~K}^{2}\right)$. Indeed the data even are in accord with the prediction that the entropy $S_{m}(T)$ approaches the high temperature limit faster than the effective moment $T \chi / C_{J}$ which in turn evolves more rapidly than $n_{f}(T)$ (see Fig. 4). Nevertheless, it is also clear that the experimental data for these quantities approach the high temperature limit considerably more slowly than predicted by the AIM theory.

Thus we have demonstrated the existence of a new low temperature scale for $\mathrm{YbAl}_{3}$ and we have shown that the crossover to local moment behavior is slower than expected based on the Anderson Impurity Model. We believe that such effects are generic to IV compounds. In our earlier work[5] on $\mathrm{YbXCu}_{4}$ we found that the slow crossover correlated with a low background conduction electron density; strong deviations occurred for $n_{c} \sim$ 0.5 /atom where $n_{c}$ was calculated from the Hall coefficient of $\mathrm{LuXC \textrm {Cu } _ { 4 }}$ in a one-band approximation. Using a similar approximation we deduce from the Hall coefficient of $\mathrm{LuAl}_{3}$ (Fig. 3a) that $n_{c} \sim 0.5 /$ atom. Hence, while the conduction electron density is not low for $\mathrm{YbAl}_{3}$, it is (in this approximation) as low as in other compounds where strong deviations from the AIM are observed.

A number of years ago we gave evidence[11] for a small coherence scale in the IV compound $\mathrm{CePd}_{3}$ based on a low temperature peak in the susceptibility and the extreme sensitivity of the transport behavior to Kondo hole impurities below 50K. A low temperature anomaly in the Hall coefficient of this and other Ce compounds also has been observed[10]. Optical conductivity measurements[2, 12, 13] showed both that the temperature scale for these effects is the same as for the renormalization of the effective mass and the onset of the hybridization gap and that the conduction electron density of $\mathrm{CePd}_{3}$ is less than 0.1 carrier/atom. Hence the anomalies appear to be associated both with the on-
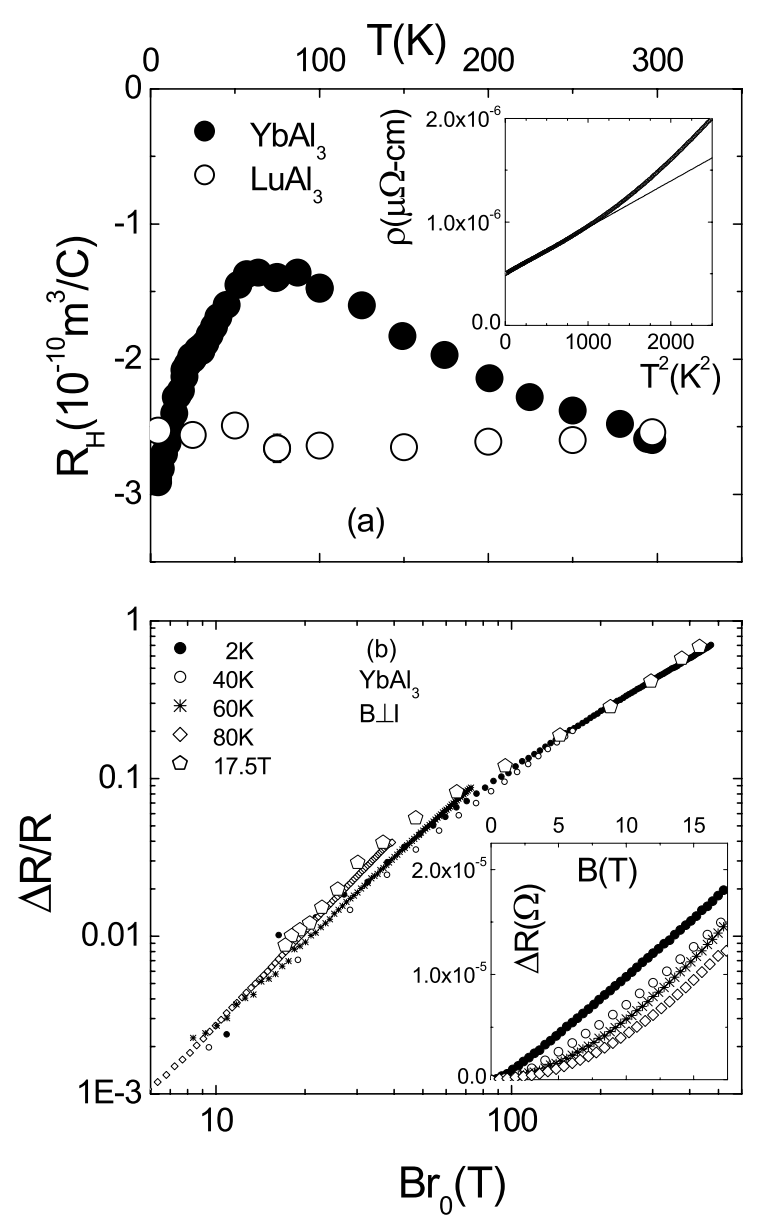

FIG. 3: a) The Hall coefficient of $\mathrm{YbAl}_{3}$ (closed circles) and $\mathrm{LuAl}_{3}$ (open circles) versus temperature. For $\mathrm{LuAl}_{3}$ a typical error bar is shown; for $\mathrm{YbAl}_{3}$ the error is smaller than the size of the symbols. Inset: the resistivity plotted versus the square of the temperature. b) Inset: The transverse magnetoresistance $\Delta R=R(H, T)-R(0, T)$ versus magnetic field for four temperatures. Main panel: The relative magnetoresistance $\Delta R / R$ versus $B r_{0}$ where $r_{0}=R(150 K / R(T))$.

set of the fully renormalized coherent ground state and with low conduction electron density. Measurement of the infrared optical conductivity is clearly a key future experiment for $\mathrm{YbAl}_{3}$.

Recent theoretical work[14, 15] on the Anderson lattice predicts both a slow crossover and a low temperature coherence scale in the limit of low conduction electron density. However, the theoretical work to date has been performed only in the Kondo limit. We have examined the extension of the slave boson mean field theory for the Anderson lattice to the case $n_{f}<1$ and $n_{c}<1$ relevant to $\mathrm{YbAl}_{3}$, where $n_{f}$ is the number of holes in the $(2 J+1)$ fold degenerate $\mathrm{f}$ level. Following Millis and Lee[16], we define the Kondo temperature $k_{B} T_{K}$ as the 


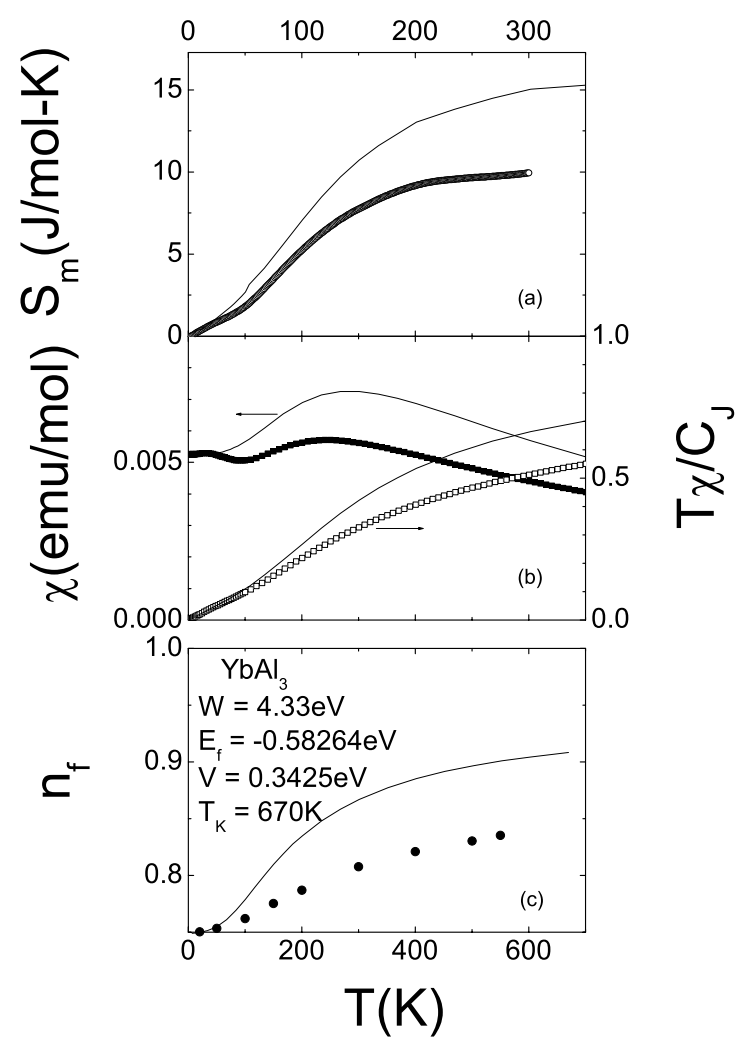

FIG. 4: (a) The $4 f$ entropy $S_{m}$, (b) the susceptibility $\chi(T)$ (solid symbols) and the effective moment $T \chi / C_{J}$ (open symbols) where $C_{J}$ is the $J=7 / 2$ Curie constant and (c) the $4 f$ occupation number $n_{f}(T)$ for $\mathrm{YbAl}_{3}$. The symbols are the experimental data and the solid lines are the predictions of the Anderson Impurity Model (AIM) calculated in the NCA with input parameters as given in the figure.

energy of the renormalized f level relative to the Fermienergy. The coherence scale is defined as the renormalized (quasiparticle) $T=0$ bandwidth which for a background conduction band density of states $\rho$ is given by $k_{B} T_{\text {coh }}=\rho \tilde{V}^{2}$ where $\tilde{V}$ is the renormalized hybridization $\tilde{V}=\sqrt{1-n_{f}} V$. In the limit $(\rho \tilde{V})^{2}<<n_{c} n_{f} /(2 J+1)^{2}$ we find that $T_{c o h} / T_{K}=n_{f} /(2 J+1)$ independent of $n_{c}$. For $\mathrm{YbAl}_{3}$ this means that $T_{c o h}$ should be an order of magnitude smaller than $T_{K}$, in qualitative agreement with the data.

In any case we assert that the two energy scales and slow crossover predicted by recent theory are features of our data, that these effects show some correlation with a standard measure of the conduction electron density and that they may be generic for IV compounds.

Work at UC Irvine was supported by UCDRD funds provided by the University of California for the conduct of discretionary research by the Los Alamos National Laboratory and by the UC/LANL Personnel Assignment Program. T. E. acknowledges the support of the Japanese Ministry of education. Work at Polytechnic was supported by DOE FG02ER84-45127. Work at Lawrence Berkeley National Laboratory was supported by DOE Contract No. DE-AC03-76SF00098. Work at Los Alamos was performed under the auspices of the DOE. The x-ray absorption experiments were performed at SSRL, which is operated by the DOE/OBES.

* Electronic address: jmlawrenc@uci.edu

[1] A. C. Hewson, The Kondo Problem to Heavy Fermions (Cambridge University Press, 1993) p. 315.

[2] B. C. Webb, A. J. Sievers and T. Mihalisin, Phys. Rev. Lett. 57, 1951 (1986).

[3] A. Hasegawa and H. Yamagami, Progress of Theoretical Physics Supplement 108, 27 (1992).

[4] N. E. Bickers, D. L. Cox and J. W. Wilkins, Phys. Rev. B 36, 2036 (1987).

[5] J. M. Lawrence, P. S. Riseborough, C. H. Booth, J. L. Sarrao, J. D. Thompson and R. Osborn, Phys. Rev. B 63, 054427 (2001).

[6] J. M. Lawrence, S. M. Shapiro, J. L. Sarrao and Z. Fisk, Phys. Rev. B 55, 14467 (1997); J. M. Lawrence, R. Osborn, J. L. Sarrao and Z. Fisk, Phys. Rev. B 59, 1134 (1999).

[7] J. L. Sarrao, C. D. Immer, Z. Fisk, C. H. Booth, E. Figueroa, J. M. Lawrence, R. Modler, A. L. Cornelius, M. F. Hundley, G. H. Kwei, J. D. Thompson and F. Bridges, Phys. Rev. B 59, 6855 (1999).

[8] A. Hiess, J. X. Boucherle, F. Givord, J. Schweizer, E. Lelievre-Berna, F. Tasset, B. Gillon and P. C. Canfield, J. Phys.: Condens. Matter 12, 829 (2000).

[9] T. Ebihara, Y. Inada, M.Murakawa, S. Uji, C. Terakura, T. Terashima, E. Yamamoto, Y. Haga, Y. Onuki and H. Harima, Journ. Phys. Soc. Japan 69, 895 (2000).

[10] E. Cattaneo, Z. Physik B 64, 305 (1986); ibid, 317.

[11] J. M. Lawrence, Y.-Y. Chen and J. D. Thompson, in Theoretical and Experimental Aspects of Valence Fluctuations and Heavy Fermions, edited by L. C. Gupta and S. K. Malik (Plenum Press, New York, 1987) p. 169.

[12] B. Bucher, Z. Schlesinger, D. Mandrus, Z. Fisk, J. Sarrao, J. F. DiTusa, C. Oglesby, G. Aeppli and E. Bucher, Phys. Rev. B 53, R2948 (1996).

[13] L. Degiorgi, F. B. B. Anders and G. Grüner, Eur. Phys. J. B 19, 167 (2001).

[14] A. N. Tahvildar-Zadeh, M. Jarrell and J. K. Freericks, Phys. Rev. B 55, R3332 (1997); Phys. Rev. Lett. 80, (1998) 5168.

[15] S. Burdin, A. Georges and D. R. Grempel, Phys. Rev. Lett. 85, 1048 (2000).

[16] A. J. Millis and P. A. Lee, Phys. Rev. B 35, 3394 (1987). 